\title{
A systematic review of patient surveillance after curative gastrectomy for gastric cancer: a brief review
}

\author{
Roberta Cardoso • Natalie G. Coburn • \\ Rajini Seevaratnam • Alyson Mahar · \\ Lucy Helyer $\cdot$ Calvin Law $\cdot$ Simron Singh
}

Received: 4 August 2011/Accepted: 9 January 2012/Published online: 2 March 2012

(C) The International Gastric Cancer Association and The Japanese Gastric Cancer Association 2012

\begin{abstract}
Background Complete resection of a gastric cancer and adjacent lymph nodes offers the only chance for cure of the disease. However, disease recurrence occurs in $22-51 \%$ of cases, and its prognosis is very poor. Many clinicians perform post-operative follow-up for these patients, although there is no consensus on the regimen, frequency of visits, mode of testing, or the rationale of a follow-up program.

Purpose The objective of this systematic review was to identify the evidence for surveillance in patients with resected gastric cancer, specifically examining the interval of follow-up and the modalities utilized.
\end{abstract}

Electronic supplementary material The online version of this article (doi:10.1007/s10120-012-0142-9) contains supplementary material, which is available to authorized users.

R. Cardoso $\cdot$ N. G. Coburn $\cdot$ R. Seevaratnam $\cdot$ A. Mahar Sunnybrook Research Institute, Sunnybrook Health Sciences Centre, Toronto, Canada

\section{N. G. Coburn $(\bowtie) \cdot$ C. Law}

Division of Surgical Oncology, Sunnybrook Health Sciences Centre, Odette Cancer Centre, Suite T2-60, 2075 Bayview Ave,

Toronto, ON M4N 3M5, Canada

e-mail: natalie.coburn@sunnybrook.ca

N. G. Coburn · L. Helyer

Department of Surgery, Dalhousie University, Halifax, Canada

N. G. Coburn · S. Singh

Department of Medical Oncology/Hematology, Sunnybrook

Health Sciences Centre and Odette Cancer Centre,

Toronto, Canada

A. Mahar

Department of Community Health and Epidemiology,

Queen's University, Kingston, Canada
Methods Electronic literature searches were conducted using Medline, Embase, and the Cochrane Central Register of Controlled Trials from January 1st 1998 to December 1st 2009. All search titles and abstracts were independently rated for relevance by a minimum of two reviewers.

Results Five articles were selected. A total of 810 patients underwent post-operative follow-up. History and physical examination, hematological and chemistry profile, endoscopy (esophagogastroduodenoscopy [EGD]), and computed tomography (CT) were the most frequently employed modalities. CT detected the majority of recurrences in the included studies. The survival post-recurrence was significantly higher in the asymptomatic group compared with symptomatic group in three studies, but this may simply reflect lead-time bias. No differences in overall survival (OS) were found.

Conclusion The included studies failed to show an improvement in OS with more intense surveillance. Further prospective studies are required to determine whether a subgroup of patients may benefit from more intensive follow-up.

Keywords Gastric cancer · Follow-up ·

Systematic review $\cdot$ Resection $\cdot$ Surveillance

\section{Introduction}

The overall survival among patients receiving curative gastrectomy unfortunately remains poor, with most cases of recurrence occurring within the first 5 years [1]. As a result, many clinicians perform post-operative follow-up or surveillance for these patients [2,3], even though there appears to be no clear consensus on the utility or mode of surveillance in these patients. 
Most guidelines aim to detect early disease recurrence and improve patient quality of life (QoL); however, they lack details on the mode, duration, and intensity of surveillance. The National Cancer Comprehensive Network (NCCN) guidelines suggest all patients should be followed after surgery, including a complete history and physical examination every 3-6 months for years 1 and 2 , every 6-12 months for years $3-5$, and then annually ongoing. Investigations are recommended as clinically indicated by symptoms. The European Society of Medical Oncology guidelines, and the British and Scottish guidelines $[4,5]$ clearly state that no evidence exists to support the notion that regular follow-up improves patient outcomes, and recommend symptom-driven visits with directed investigations only in patients who are candidates for further treatment. It is reasonable to hypothesize that patients may experience benefit by postsurgical surveillance, if early detection of recurrence prompts interventions that may lead to a survival advantage and/or an increase in QoL. The objectives of our systematic review were to assess and update the current literature with a systemic review of follow-up in resected gastric cancer.

\section{Methods}

Data sources

Electronic literature searches were conducted using Medline and Embase from January 1st 1998 to May 1st 2011 according to the search algorithm outlined in Appendix 1 of the electronic supplementary material (ESM). A separate search of the Cochrane Central Register of Controlled Trials (1998-2009) was performed using the search term "gastric cancer" (Appendix 1 of the ESM). No attempt was made to locate unpublished material.

Study selection and review process

All eligible studies had to meet the following criteria: (1) included patients with gastric adenocarcinoma, (2) assessed outcomes of follow-up after gastrectomy, (3) published in a peer-reviewed journal, and (4) in the English language. Studies were excluded according to the following criteria: reviews, meta-analyses, systematic reviews, abstracts, editorials or letters, case reports, and guidelines. All electronic search titles, selected abstracts and full-text articles were independently reviewed by a minimum of two reviewers (NC, RC, and RS). Reference lists from review papers and relevant articles were also examined for additional studies. Disagreements on study inclusion/exclusion were resolved with a consensus meeting.
Data extraction

A systematic approach to data extraction was used to produce a descriptive summary of study characteristics, interventions, and study findings. The following data were collected: study characteristics (country and design), patients' characteristics (gender, age, tumor location, and stage), follow-up regimens (period and modalities), and outcomes (modalities, detection rates, recurrence rates, and survival). The first reviewer (RC) independently extracted the data and a second reviewer (NC, RS) reviewed the data extraction. No attempt was made to contact authors for additional information. When necessary, data was extracted or estimated from survival curves.

\section{Results}

A total of 3608 abstracts were identified for preliminary review; 13 articles were reviewed and five were selected for data abstraction (Appendix 2 of the ESM). Appendix 3 of the ESM shows the characteristics of the five included studies and the 810 patients enrolled in these studies. Among the five articles, none were randomized controlled trials (RCTs) or prospective studies.

\section{Surveillance modalities (Appendix 3 of the ESM)}

A range of modalities utilized to follow patients was identified: history and physical examination, abdominal ultrasonography (US), computed tomography (CT), endoscopy (esophagogastroduodenoscopy [EGD]), endoscopic ultrasound (EUS), chest radiography, blood count, chemistry profile, tumor markers, barium enema, and bone scintigraphy. However, only two studies reported results on the types of modalities that detected the recurrence (Appendix 3 of the ESM).

\section{Survival (Table 1)}

Disease-free survival (DFS) from the original operation to first recurrence was reported by all five studies. Only Tan et al. reported that DFS was significantly shorter in the intensive follow-up group compared to the regular followup group (11.5 vs. 19.2 months, $P=0.02$ ) [2].

Survival post-recurrence (SPR) was provided by four of the five studies of interest. All four studies [3, 6-8] reported a statistically significant improvement in postrecurrence survival for the asymptomatic groups. Overall survival (OS) was only reported by Kodera et al. [8] and Tan et al. [2]. The study by Kodera's group [8] included OS curves with an estimated median OS of 40 months for symptomatic patients and 51.7 months for asymptomatic 
Table 1 Recurrence and survival

\begin{tabular}{|c|c|c|c|c|c|c|c|c|c|c|c|}
\hline Author & No. of pts & $P$ & $\operatorname{RE}(N)$ & DSS & $P$ & DFS & $P$ & SPR & $P$ & OS & $P$ \\
\hline \multirow[t]{2}{*}{ Bennett [6] } & $\mathrm{AG}=99$ & $<0.0001$ & 99 & 29.4 & $<0.05$ & 10.8 & NS & 13.5 & $<0.01$ & - & - \\
\hline & $\mathrm{SG}=283$ & & 283 & 21.6 & & 12.4 & & 4.8 & & - & \\
\hline \multirow[t]{2}{*}{ Bohner [7] } & $\mathrm{AG}=15^{\mathrm{a}, \mathrm{b}, \mathrm{c}}$ & NS & 15 & - & - & $13(4-62)$ & - & $7^{\mathrm{d}}$ & 0.017 & & - \\
\hline & $\mathrm{SG}=52^{\mathrm{c}, \mathrm{e}, \mathrm{f}}$ & & 52 & - & & $13(2-75)$ & & 5 & & - & \\
\hline \multirow[t]{2}{*}{ Kodera [8] } & $\mathrm{AG}=88^{\mathrm{g}}$ & NR & 88 & - & - & $30^{\mathrm{h}}$ & 0.2 & $29^{\mathrm{h}}$ & $<0.0001$ & $51.7^{\mathrm{h}}$ & 0.19 \\
\hline & $\mathrm{SG}=109^{\mathrm{i}}$ & & 109 & - & & $30^{\mathrm{h}}$ & & $25^{\mathrm{h}}$ & & $40^{\mathrm{h}}$ & \\
\hline Mikani [3] & $62^{\mathrm{j}, \mathrm{k}}$ & NA & 62 & - & - & 22.2 & - & $\begin{array}{l}\mathrm{SG}=13.74^{\mathrm{h}} \\
\mathrm{AG}=27.5^{\mathrm{h}}\end{array}$ & 0.0011 & - & - \\
\hline \multirow[t]{2}{*}{ Tan [2] } & $\mathrm{IF}=49$ & $<0.01$ & 24 & - & - & Mean $=11.5 \pm 2.1$ & 0.02 & - & - & 49.2 & 0.46 \\
\hline & $\mathrm{RF}=53$ & & 23 & - & & Mean $=19.2 \pm 2.7$ & & - & & 45.6 & \\
\hline
\end{tabular}

$A G$ asymptomatic group, $D F S$ disease-free survival, $D S S$ disease-specific survival, $N A$ not appropriate, $N R$ not reported, $N S$ not statistically significant, $O S$ overall survival, $P$ significance, $R E$ recurrence, $S G$ symptomatic group, $S P R$ survival post-recurrence

a 6 patients received adjuvant chemotherapy

b 4 patients received adjuvant radiation

c Multimodal treatment possible

d One patient surviving $>5$ years after treatment was excluded

e 3 patients received adjuvant chemotherapy

f 8 patients received adjuvant radiation

g 74 patients received chemotherapy after recurrence

h Estimated from curves

i 79 patients received chemotherapy after recurrence

j 9 patients underwent surgery after recurrence

k 30 patients received chemotherapy after recurrence

patients $(P=0.19)$. Tan et al. reported an average OS of 4.1 years (49.2 months) in the intensive follow-up group and 3.8 years (45.6 months) in the regular follow-up group $(P=0.46)$ [2]. Bennett's group reported overall diseasespecific survival (DSS) from curative gastrectomy to death [6] of 29.4 months for asymptomatic patients and 21.6 months for symptomatic patients $(P<0.05)$.

\section{Discussion}

Our systematic review found a lack of evidence supporting follow-up for curatively resected patients. The limited data is retrospective, with bias and confounding preventing definitive conclusions. Although the study by Bennett's group [6] reported an overall disease-specific (DSS) survival advantage with regular follow-up, the impact of detecting asymptomatic recurrences could not be separated from other important biological factors, such as advanced stage, poor differentiation, short disease-free interval, and multiple sites of recurrence on multivariate analysis. Thus, biological factors are likely the predominant feature when considering post-recurrence outcomes, rather than early detection of asymptomatic recurrences. No other study was able to demonstrate an improvement in OS associated with follow-up. Although most of the studies did show an increase in post-recurrence survival in the asymptomatic group of patients, this likely reflects lead-time bias, in which the observed prolonged survival is due to earlier detection of recurrence, rather than being due to a true effect on disease outcome. Additionally, patients who present with symptomatic recurrences likely have a heavier burden of disease or biologically more aggressive disease.

Only one study examined intensive versus regular follow-up, and the authors found no difference in the OS [2], although recurrences were detected earlier in the intensive group. However, due to the retrospective non-randomized nature of this study, it is likely that those patients who received more intensive follow-up presented with more aggressive disease and increased symptoms of recurrence and as a result were investigated to a greater degree. This confounding factor makes the interpretation of the results and any recommendations problematic.

We were unable to determine any superior mode or frequency of surveillance. A range of modalities was used to follow patients, but the study by Tan's group [2] found that CT was the modality responsible for detection of the majority of the recurrences (60\%). Other studies showed that CT and EUS may also be beneficial in recurrence detection [7], especially among symptomatic patients. 
Tumor markers appear to be an easy test to perform in order to detect recurrences, but they are neither highly sensitive, nor specific, and cannot localize the recurrence site [9].

Detection of early recurrence may theoretically allow patients to receive palliative treatments such as chemotherapy, resulting in better outcomes. In the study by Kodera's group, detection of recurrent disease at an asymptomatic stage enabled a greater proportion of patients to be treated with chemotherapy $(P=0.076)$, possibly because performance status was better at the time of detection [8]. Clearly, biology is a dominant factor when assessing for recurrence in gastric cancer. In the study by Mikani et al. [3], patients with hematological metastases, loco-regional recurrence, and recurrence in the remnant stomach responded well to systemic therapy. With improved systemic treatments for gastric cancer utilizing newer agents such as trastuzumab [10], it may be possible that early detection of asymptomatic recurrences will allow for earlier and more effective treatment. Future RCTs should be designed to identify specific patients in whom early recurrence detection and intervention may result in improved outcomes.

An important omission in the current literature is QoL. It is unclear whether ongoing surveillance will improve or reduce patient QoL and none of the included studies evaluated this. Importantly, benign post-surgical complications, nutritional disorders, and the need for psychosocial support can be detected during follow-up visits. However, the effects of return visits, extra diagnostic tests, and the stress associated with them must be also assessed. Studies examining routine follow-up of colorectal cancer patients $[11,12]$ have reported mixed effects on QoL measures. A study of patients with various cancers reported that a majority of patients favor regular follow-up despite the disadvantages [5].

\section{Conclusion}

Although follow-up is common in gastric cancer care, there is no evidence to suggest that it has any survival benefit, and no studies measured QoL. Limitations in study design, a lead-time bias, and the predominant effect of aggressive biological features on outcomes are likely explanations of this lack of survival benefit. While it is still possible that a proportion of patients may benefit from routine post-gastrectomy follow-up, prospective randomized controlled trials would be necessary to demonstrate such an effect and the subgroups in which a benefit may be found.
Acknowledgments This study was funded by the Canadian Cancer Society (Grant \# 019325). Dr. Coburn is supported by a Ministry of Health and Long Term Care Career Scientist Award. Dr. Law is supported by the Hanna Family Research Chair in Surgical Oncology.

\section{References}

1. Marrelli D, De Stefano A, de Manzoni G, Morgagni P, Di Leo A, Roviello F. Prediction of recurrence after radical surgery for gastric cancer: a scoring system obtained from a prospective multicenter study. Ann Surg. 2005;241(2):247-55.

2. Tan IT, So BYJ. Value of intensive follow-up of patients after curative surgery for gastric carcinoma. J Surg Oncol. 2007;96:503-6.

3. Mikani K, Yamashita Y, Maekawa T, Shinohara T, Yamauchi Y, Hoshino $\mathrm{S}$, et al. Surveillance program for recurrence after curative gastric cancer surgery. Chir Gastroenterol. 2007; 23:392-8.

4. Jackson C, Cunningham D, Oliveira J, On behalf of the ESMO Guidelines Working Group. Gastric cancer: ESMO clinical recommendations for diagnosis, treatment and follow-up. Ann Oncol. 2009;20(Supplement 4):iv34-6.

5. Allum WH, Griffin SM, Watson A, Colin-Jones D. Guidelines for the management of oesophageal and gastric cancer. Gut. 2002;50(Suppl 5):v1-23.

6. Bennett JJ, Gonen M, D'Angelica M, Jaques DP, Brennan MF, Coit DG. Is detection of asymptomatic recurrence after curative resection associated with improved survival in patients with gastric cancer? J Am Coll Surg. 2005;201(4):503-510.

7. Bohner H, Zimmer T, Hopfenmuller W, Berger G, Buhr H-J. Detection and prognosis of recurrent gastric cancer-is routine follow-up after gastrectomy worthwhile? Hepatogastroenterology. 2000;47:1489-94.

8. Kodera Y, Ito S, Yamamura Y, Mochizuki Y, Fujiwara M, Hibi $\mathrm{K}$, et al. Follow-up surveillance for recurrence after curative gastric cancer surgery lacks survival benefit. Ann Surg Oncol. 2003;10(8):898-902.

9. Qui MZ, Lin JZ, Wang ZQ, Pan ZZ, Luo HY, Li YH, et al. Cutoff value of carcinoembryonic antigen and carbohydrate antigen 19-9 elevation levels for monitoring recurrence in patients with resectable gastric adenocarcinoma. Int $\mathrm{J}$ Biol Markers. 2009;24(4):258-64.

10. Bang YJ, Cutsem EV, Feyereislova A, Chung HC, Shen L, Sawaki A, et al. Trastuzumab in combination with chemotherapy versus chemotherapy alone for treatment of HER2-positive advanced gastric or gastro-oesophageal junction cancer (ToGA): a phase 3, open-label, randomised controlled trial. Lancet. 2010;376(9741):687-97.

11. Wattchow DA, Weller DP, Esterman A, Pilotto LS, McGorm K, Hammett Z. General practice vs surgical-based follow-up for patients with colon cancer: randomised controlled trial. $\mathrm{Br} \mathrm{J}$ Cancer. 2006;94:1116-21.

12. Stiggelbout AM, de Haes JC, Vree R, van de Velde CJ, Bruijninckx CM, van Groningen K. Follow-up of colorectal cancer patients: quality of life and attitudes towards follow-up. $\mathrm{Br} \mathrm{J}$ Cancer. 1997;75(6):914-20. 